\title{
Simulation Research on Dynamic Matrix Control of Middle Speed Mill Shihe $\mathrm{CHEN}^{1}$, Xi ZHANG ${ }^{1}$, Shubin Xiao ${ }^{2}$, Peng $\mathrm{Liao}^{2}$, Haihua Xiao ${ }^{3}$, Jianguo DUAN ${ }^{4}$, Li ZHANG ${ }^{4}$ Yuguang NIU ${ }^{4}$, Zhongwei LIN ${ }^{4, a}$ \\ ${ }^{1}$ Electric Power Research Institute of Guangdong Power Grid Corporation Guangzhou, 510000,China \\ ${ }^{2}$ Changsha University of Science and Technology, Changsha, 410114, China \\ ${ }^{3}$ Guangxi University, Nanning, 530004, China \\ ${ }^{4}$ School of control \& computer engineering North China Electric power University Beijing, 102206, China \\ aemail:1531920937@QQ.COM
}

Keywords: Thermal Power Plant; Dynamic Matrix Control; Middle Speed Mill; Dynamic Simulation

\begin{abstract}
By using the on-site parameters from the middle speed mill (MSM) of the power plant, the mathematical model of middle speed mill is given by using the test-modeling method. Based on the obtained model, the dynamic matrix control (DMC) of middle speed mill is introduced in MATLAB/SIMULATION environmental. The impact of the parameters of the DMC controller on the final outputs of MSM have been widely studied, such as model mismatch, variations of the prediction and control horizons, and interruption time. The simulations are performed in comparison with the convention proportion integration differentiation (PID) controller. The results show that the introduced DMC provides a desired performance.
\end{abstract}

\section{Introduction}

With continuing development of the electric power system, the capacity of thermal power unit is larger and larger. The middle speed mill has been widely used for less occupied area, low energy consumption, and simplified powder process. The SMS is a nonlinear, coupled and multivariable system, which is difficult to obtain the acceptable performance by using conventional control schemes.

For their reliability and simplicity, the conventional PID controller is well accepted in the engineering field, and it also has been widely used in power systems. However, the parameters of the conventional PID controller are usually tuned with the approximated linearized model. In this situation, there must be a compromise between the control performance and robustness. The model predictive control (MPC) has been deeply researched and widely used in the industrial fields. DMC, a kind of non-parametric model based MPC, is designed based on the step response model which is easy to be obtained. For predictive model, horizon optimization and feedback correction, DMC has a good robustness and can be used to control the system without the accurate model. Ref. [1] mainly studied the DMC control of the furnace feedwater control system. Ref. [2] studied the application of predictive control about the boiler feedwater control system. Ref. [3] mainly analyzed the current situation of the predictive control system in the electric power plant; Ref. [4] studied the application of predictive control in the system of overheating temperature control system; Ref. [5] studied the application of RBF neural network information fusion technology in the meddle speed mill; Ref. [6] studied how to adopt field data to build the dynamic mathematical model about the middle mill; From the view point of the middle speed mill, Ref. [7] builds the mathematical model .

In this paper, the dynamic matrix control algorithm is a kind of predictive control algorithm based on the step response of the object, and it is suitable for the non-minimum phase system with time delay and open loop asymptotic stability. Simulation experiment indicates that the dynamic matrix prediction control is applied to the middle speed mill, possessing applicable value. 


\section{Dynamic State Characteristics of the Controlled Object of the Middle Speed Mill}

The adjusting section of the middle speed mill is the maintenance of the mill outlet temperature and the temperature is equal to specified value, ensuring mill output, so, its model can be seen as controlled object of a double-input and double-output. According to the field measurement and the step response curve in the milled speed mill of power plant, the scheme adopt test modeling method. Through per-unit, mathematical model is

$$
\left[\begin{array}{c}
T \\
N
\end{array}\right]=\left[\begin{array}{ll}
G_{T h}(s) & G_{T C}(s) \\
G_{N h}(s) & G_{N C}(s)
\end{array}\right]\left[\begin{array}{l}
\mu_{h} \\
\mu_{C}
\end{array}\right]
$$

\section{Middles Speed Mill Dynamic Matrix Control Algorithm}

Dynamic matrix control algorithm what is based on step response model includes mainly predictive model, feedback compensation and rolling optimization. The various parameters is respectively.

$$
\vec{Y}=\left[\begin{array}{ll}
T & N
\end{array}\right]^{T}, \vec{Y}_{s}=\left[\begin{array}{ll}
T_{s} & N_{s}
\end{array}\right]^{T}, \stackrel{\mathrm{V}}{U}=\left[\begin{array}{ll}
\mu_{h} & \mu_{c}
\end{array}\right]^{T} \stackrel{\mathrm{V}}{Y_{r}}=\left[\begin{array}{ll}
T_{r} & N_{r}
\end{array}\right]^{T}, \vec{D}=\left[\begin{array}{ll}
T_{D} & N_{D}
\end{array}\right]^{T}, \vec{Y}_{m}=\left[\begin{array}{ll}
\widehat{T} & \widehat{N}
\end{array}\right]^{T}
$$

\section{A.Prediction Model}

Prediction model is for getting the predicted value of the future output of the object, the mathematical expression is.

$$
\vec{Y}_{m}(k+1)=A \Delta \vec{U}(k)+\vec{Y}_{0}(k+1)
$$

In the equation (2), Unit A is represent as: $A=\left[\begin{array}{ll}A_{11} & A_{12} \\ A_{21} & A_{22}\end{array}\right]$, which is a dynamic matrix.

And still it can be give as:

$$
\begin{aligned}
& A_{11}=\left[\begin{array}{cccc}
)_{1}^{T h} & \mathrm{~L} & \mathrm{~L} & 0 \\
\boldsymbol{a}_{2}^{T h} & )_{1}^{T h} & & \mathrm{M} \\
\mathrm{M} & & \mathrm{O} & \mathrm{M} \\
\mathrm{M} & & & )_{\boldsymbol{a}_{1}^{T h}}^{T h} \\
\hat{\boldsymbol{a}}_{P}^{T h} & \mathrm{~L} & \mathrm{~L} & \boldsymbol{a}_{P-M+1}^{T h}
\end{array}\right] \quad A_{12}=\left[\begin{array}{cccc}
\hat{a}_{1}^{N h} & \cdots & \cdots & 0 \\
\widehat{a}_{2}^{N h} & \widehat{a}_{1}^{N h} & & \vdots \\
\vdots & & \ddots & \vdots \\
\vdots & & & \widehat{\boldsymbol{a}}_{1}^{N h} \\
\widehat{a}_{P}^{N h} & \cdots & \cdots & \widehat{a}_{P-M+1}^{N h}
\end{array}\right] \\
& A_{21}=\left[\begin{array}{cccc}
\hat{a}_{1}^{T c} & \cdots & \cdots & 0 \\
\hat{a}_{2}^{T c} & \widehat{a}_{1}^{T c} & & \vdots \\
\vdots & & \ddots & \vdots \\
\vdots & & & \hat{a}_{1}^{T c} \\
\widehat{a}_{P}^{T c} & \cdots & \cdots & \widehat{a}_{P-M+1}^{T c}
\end{array}\right] \quad A_{22}=\left[\begin{array}{cccc}
\hat{a}_{1}^{N c} & \cdots & \cdots & 0 \\
\hat{a}_{2}^{N c} & \widehat{a}_{1}^{N c} & & \vdots \\
\vdots & & \ddots & \vdots \\
\vdots & & & \hat{a}_{1}^{N c} \\
\widehat{a}_{P}^{N c} & \cdots & \cdots & \widehat{a}_{P-M+1}^{N c}
\end{array}\right]
\end{aligned}
$$

\section{B.Feedback Compensation}

On the basis of predicted model output, the predicted value of system output is revised by the feedback of actual output error, realizing closed-loop prediction. That is

$\vec{Y}_{c}(k+1)=\vec{Y}_{m}(k+1)+\vec{g}_{0}\left[\vec{Y}(k)-\vec{Y}_{m}(k)\right]$

The prediction output after the feedback compensation is

$$
\begin{aligned}
& \vec{Y}_{c}(k+1)=\left[\begin{array}{ll}
\vec{T}_{c}(k+1) & \vec{N}_{c}(k+1)
\end{array}\right]^{T} ; \\
& \vec{T}_{c}(k+1)=\left[\begin{array}{lll}
T_{c}(k+1) \cdots T_{c}(k+P)
\end{array}\right]^{T} ; \\
& \vec{N}_{c}(k+1)=\left[\begin{array}{llll}
N_{c}(k+1) \cdots N_{c}(k+P)
\end{array}\right]^{T} \vec{g}_{0}=\operatorname{diag}\left[\begin{array}{llll}
1 & 1 & \cdots & 1
\end{array}\right]
\end{aligned}
$$




\section{Horizon Optimization}

Dynamic matrix control algorithm adopt horizon optimization performance index. It is:

$$
J=\left\|\vec{Y}_{r}(k+1)-\vec{Y}_{c}(k+1)\right\|_{\vec{Q}}^{2}+\|\Delta \vec{U}(k)\|_{\vec{R}}^{2}
$$

The error weighted matrix and the controlling variable weighted matrix are given below:

$\vec{Q}=\operatorname{diag}\left\{\begin{array}{llllllll}q_{T 1}^{2} & q_{T 2}^{2} & \cdots & q_{T P}^{2} & q_{N 1}^{2} & q_{N 2}^{2} & \cdots & q_{N P}^{2}\end{array}\right\}$

$\vec{R}=\operatorname{diag}\left\{\begin{array}{llllllll}r_{T 1}^{2} & r_{T 2}^{2} & \cdots & r_{T M}^{2} & r_{N 1}^{2} & r_{N 2}^{2} & \cdots & r_{N M}^{2}\end{array}\right\}$

Assuming that the softness parameter of mill outlet temperature and primary air flow of mill are and , thus, reference trajectory is:

$\stackrel{\mathrm{V}}{Y_{r}}(k+1)=\Lambda_{2} Y_{s}(k+1)+\Lambda_{1} Y(k+1)$

Optimal control law is:

$\Delta \vec{U}(k)=\left(A^{T} \vec{Q} A+R\right)^{-1} A^{T} \vec{Q}\left\{\Lambda\left[\vec{Y}_{s}(k+1)-\vec{Y}(k+1)\right]-\vec{p}(k+1)\right\}$

\section{Simulation of the Medium Speed Mill Dynamic Matrix Control}

In the paper, calling predictive control toolbox function of the Matlab so that realize middle speed mill dynamic matrix control simulation. The length of the predicted time-domain is 6 seconds; The length of the controlling time-domain is 2 seconds; End time of step is 90 seconds; Sampling period is 3 seconds; The output error weighted matrix is Q; The input control weighted matrix is R; Middle speed mill outlet temperature and step input of mill load are 20\%; The simulation time is 100 seconds; The simulation curve of the input and output what are unconstrained is shown in figure 1.

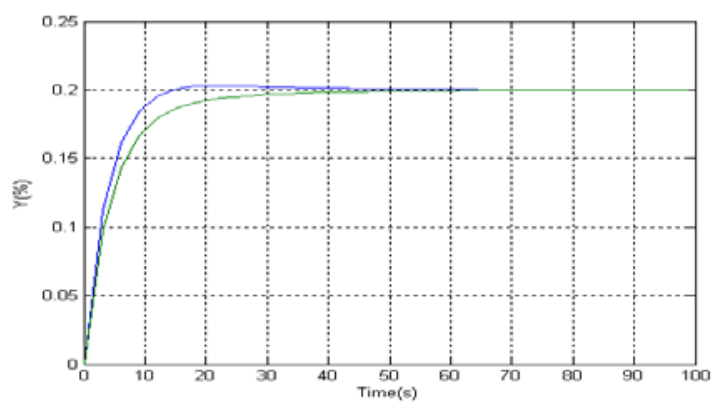

Fig.1. Simulation curve of the dynamic matrix control.

From the simulation curve of figure 1, we can see that middle speed mill outlet temperature meet non-overshoot; Adjusting time is about 30 seconds; Meanwhile, the overshoot of mill load is less than 2\%; Adjusting time is about 13 seconds; Both regulation quality meet the engineering requirements.

\section{A.The Simulation of Robustness Parameters Change of the Mill Object}

We only change the time constant of the controlled object under the other simulation conditions unchanged. Through a large number of simulation experiments, thus, the range of time constant for the controlled object is:

$$
\begin{gathered}
T_{T h} \geq 6.4 \mathrm{~s}, \quad T_{T C} \geq 4.8 \mathrm{~s}, \\
T_{N h} \geq 1.6 \mathrm{~s}, \quad T_{N C} \geq 2 \mathrm{~s}
\end{gathered}
$$

While the time constant of the controlled object take the minimum value, the simulation curve is shown in figure 2 . 


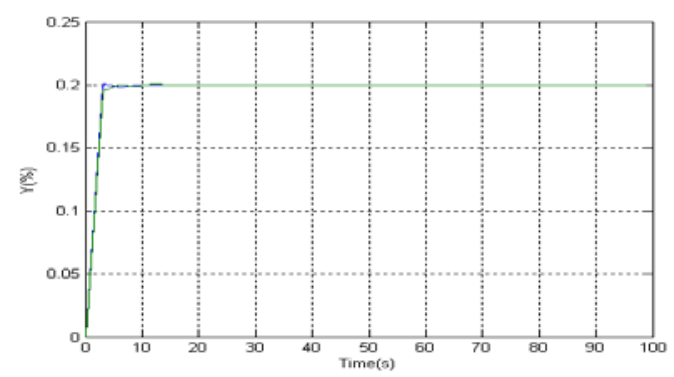

Fig.2. The simulation curve of robustness of mill object.

The simulation results shows that the time constant, all performance indexes meet the requirements. The experiments showed that even though the time constant of the controlled object is changed in a certain range, the control quality of the system is still in accordance with the engineering requirement. This shows that the design of the medium speed mill dynamic matrix control system has good robustness.

\section{B.The Simulation for the Variations of Prediction and Control Horizons}

The transfer function of the middle speed mill is the same used before, while the optimal values of sampling period and end time are adopted here. It can be assumed that the final time is 90 , and delter is 3 . When the length of the prediction horizon $\mathrm{P}$ is 30 and the length of the control horizon $\mathrm{M}$ is 2, the quality control was the best. The simulation curve is shown in figure 3.

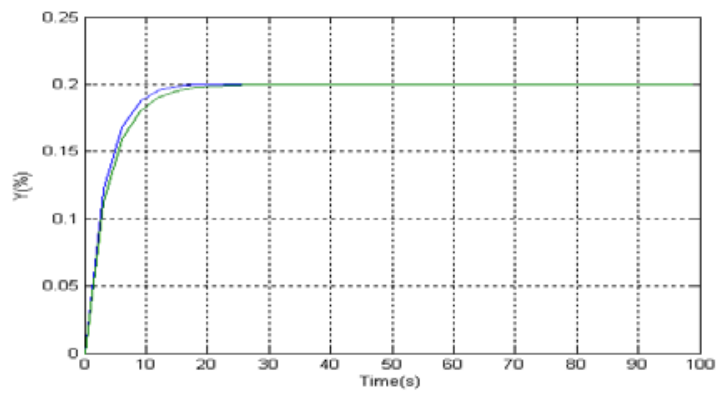

Fig.3. The simulation curve of the length of the predicted time-domain and the length of the controlling time-domain

From the simulation curve of figure 3 . We can see that the output result is good and to achieve a stable state for 20 seconds. However, increasing time-domain unceasingly, we can see that the curve effect improvement is not obvious. This parameter is the best value.

\section{Selection of the Parameters of the Output Error Weighted Matrix and theCcontrolling Variable Weighted Matrix}

The error weighted matrix and the controlling variable weighted matrix make an more influence on curve stability and response speed. After several simulation, the output error weighted matrix of quadratic performance index can be taken for , and the controlling variable weighted matrix of quadratic performance index is. The simulation result is shown in figure 4 . 


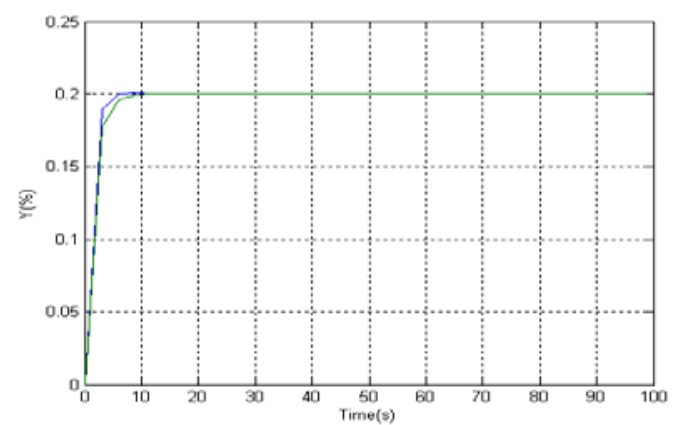

Fig.4. The output error weighted matrix and the controlling variable weighted matrix under the given numerical simulation

From the figure 4, it is seen that the output overshoot is very fine and the system is stable at $\mathrm{t}=10 \mathrm{~s}$, which has the same dynamic performance as the ideal system.

At the moment, if we further increase the numerical value of the error weighted matrix, or reduce the parameters of the controlling variable weighted matrix, the adjusting time will reduce further in small amplitude. However, the system going into the process of steady state is not excessive and does not accord with the actual industrial process.

\section{Comparison With Control Scheme of Convention PID}

The PID controlling scheme of the middle speed mill consists of two relatively independent and feed-forward single-loop control system. That is the control loop of the outlet temperature of mill and control loop of mill load with feed-forward. The best parameters of the PID controller are $K_{p}=2, K_{i}=0.01, K_{d}=80 \quad$ by a lot of simulation experiments; The parameters of mill load controller are $K_{p}=4, K_{i}=0.02, K_{d}=90$. The best response curve is shown in figure 5 .

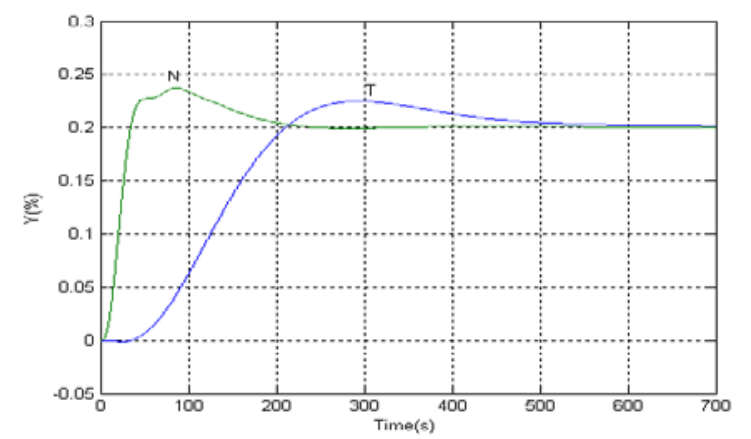

Fig.5. The output error weighted matrix and the controlling variable weighted matrix under the given numerical simulation.

From figure 5,the overshoot of the outlet temperature of mill is $12.5 \%$,and the adjusting time is 420 s; The overshoot of mill load is $19 \%$, adjusting time is 170 s.

In this paper, from the robustness and disturbance performance of the control system, we can compare the conventional PID control with the dynamic matrix control simulation, and move on to the simulation. A large number of simulation experiments show that the design of the dynamic matrix control scheme is superior to conventional PID control.

\section{Conclusion}

Middle speed mill is a intercoupling complicated controlled object, and it is a control system of a two-input and two-output.In this paper, middle speed mill dynamic matrix control scheme is presented, according to the theory of the dynamic matrix prediction control. The main characteristic of this scheme is that the paper solves successfully the control problem of middle speed mill and control index is superior to the conventional PID control scheme through prediction model, feedback compensation, and rolling optimization . 


\section{References}

[1] G. Eason, B. Noble, and I. N. Sneddon, "On certain integrals of Lipschitz-Hankel type involving products of Bessel functions,” Phil. Trans. Roy. Soc. London, vol. A247, pp. 529-551, April 1955. (references)

[2] J. Clerk Maxwell, A Treatise on Electricity and Magnetism, 3rd ed., vol. 2. Oxford: Clarendon, 1892, pp.68-73.

[3] I. S. Jacobs and C. P. Bean, "Fine particles, thin films and exchange anisotropy," in Magnetism, vol. III, G. T. Rado and H. Suhl, Eds. New York: Academic, 1963, pp. 271-350.

[4] K. Elissa, “Title of paper if known,” unpublished.

[5] R. Nicole, “Title of paper with only first word capitalized,” J. Name Stand. Abbrev., in press.

[6] Y. Yorozu, M. Hirano, K. Oka, and Y. Tagawa, "Electron spectroscopy studies on magneto-optical media and plastic substrate interface,” IEEE Transl. J. Magn. Japan, vol. 2, pp. 740-741, August 1987 [Digests 9th Annual Conf. Magnetics Japan, p. 301, 1982].

[7] M. Young, The Technical Writer’s Handbook. Mill Valley, CA: University Science, 1989.

[8] Electronic Publication: Digital Object Identifiers (DOIs):

Article in a journal:

[9] D. Kornack and P. Rakic, "Cell Proliferation without Neurogenesis in Adult Primate Neocortex,” Science, vol. 294, Dec. 2001, pp. 2127-2130, doi:10.1126/science.1065467.

Article in a conference proceedings:

[10]H. Goto, Y. Hasegawa, and M. Tanaka, "Efficient Scheduling Focusing on the Duality of MPL Representatives,” Proc. IEEE Symp. Computational Intelligence in Scheduling (SCIS 07), IEEE Press, Dec. 2007, pp. 57-64, doi:10.1109/SCIS.2007.357670. 